\title{
A NOTE ON A THEOREM OF WEYL
}

KENNETH K. WARNER ${ }^{1}$

In 1909, H. Weyl [7] proved that for a selfadjoint operator in Hilbert space, perturbation by addition of a selfadjoint compact operator leaves an "essential" part of the spectrum invariant (namely, the limit points of the spectrum and the points of infinite multiplicity). Coburn [1] has given an extension of this theorem to hyponormal operators. Nieto [3] has obtained a generalization of the theorem to a class of operators in a Banach space. The purpose of this note is to give another interpretation of the theorem of Weyl. Hyponormal and symmetrizable operators satisfy this theorem of Weyl-type. However, these results are apparently weaker than those of Coburn and Nieto. The connection with Coburn's theorem is established.

Let $T$ be a bounded linear operator on a Banach space $X$. Define the nullity, $n(T)$, as the dimension of $N(T)=\{x: T x=0\}$ and the defect, $d(T)$, as the dimension of $X / R(T)$, where $R(T)$ is the range of $T$. The index of $T$ is given by $k(T)=d(T)-n(T)$. Let $B(X)$ denote the bounded linear operators on $X, \mathfrak{e}(X)$ the compact linear operators. Let $\sigma(T)$ be the spectrum of the operator $T$. After Coburn [1], we define the Weyl spectrum by

$$
\omega(T)=\bigcap_{c \in \mathbf{e}_{(X)}} \sigma(T+C)
$$

Schechter [4] has shown that the complement of $\omega(T), C \omega(T)$ $=\{\lambda: k(\lambda-T)=0$, and $d(\lambda-T)<\infty$ and $n(\lambda-T)<\infty\}$. Let $\alpha(T)$ denote the ascent of $T$; that is, the smallest nonnegative such that the null spaces $N\left(T^{n}\right)$ are equal if $n \geqq \alpha(T)$. The descent $\delta(T)$ is the smallest integer such that the ranges $R\left(T^{n}\right)$ are equal if $n \geqq \delta(T)$. Finally, if $\lambda_{0}$ is an isolated point of $\sigma(T)$ that is a pole of the resolvent operator $(\lambda-T)^{-1}$, then $\lambda_{0}$ is a pole of the resolvent of finite rank if the spectral projection associated with $\left\{\lambda_{0}\right\}$ has finite dimensional range (see Taylor [5], [6]).

The following gives a general condition for a theorem of Weyl type to hold.

THEOREM. If the ascent of $\lambda-T$ is finite for a dense set of points $\lambda$, then $\omega(T)$ consist of $\sigma(T)$ except for poles of the resolvent of finite rank.

Received by the editors November 22, 1968.

1 I wish to thank the referee for his helpful suggestions. 
Proof. Since $\omega(T)$ is the intersection of closed sets, then its complement $C \omega(T)$ is open. If $\lambda \in C \omega(T)$, then $n(\lambda-T)=d(\lambda-T)<\infty$. If $\lambda_{0} \in C \omega(T) \cap \sigma(T)$, then there is an open connected set $S$ about $\lambda_{0}$ such that $n(\lambda-T)=d(\lambda-T)$ for $\lambda \in S$. For each $\mu \in S$ with finite ascent, we conclude, by Theorem 4.5 of Taylor [5], that $\alpha(\mu-T)$ $=\delta(\mu-T)$. Now, Theorem 9.4 of Taylor [5] implies that $\mu$ is an isolated point of $\sigma(T)$. Thus, $S \cap \rho(T) \neq \varnothing$, where $\rho(T)$ is the resolvent set for $T$. Theorem 9.6 of Taylor [5] implies that $S \cap \sigma(T)$ consists of isolated points of $\sigma(T)$ that correspond to poles of the resolvent, $(\lambda-T)^{-1}$, of finite rank. Thus $\sigma(T) \cap C \omega(T)$ consists of isolated points such that each point is a pole of finite rank of the resolvent.

If $\lambda$ is an isolated point of $\sigma(T)$ that is a pole of the resolvent of finite rank, then $n(\lambda-T)=d(\lambda-T)<\infty$ by Theorem 9.2 of Taylor [5]. Consequently, $\lambda \notin \omega(T)$ by Schech ter's characterization of $C \omega(T)$.

CoRollary 1. If the spectrum of $T$ is a nowhere dense set, then $\omega(T)$ consists of $\sigma(T)$ except for poles of the resolvent of finite rank.

The result analogous to Coburn's [1] generalization to hyponormal operators of the Weyl theorem depends on the following lemma. An operator is said to be hyponormal if $A^{*} A \geqq A A^{*}$.

Lemma 1. If $A$ is a hyponormal operator on a Hilbert space, then $A$ has ascent 0 or 1 .

Proof. Let $x \in N\left(A^{2}\right)$. Then

$$
\begin{aligned}
\left(\left(A^{*} A\right)^{2} x, x\right) & =\left(A A^{*} A x, A x\right) \\
& \leqq\left(A^{*} A A x, A x\right)=0,
\end{aligned}
$$

since $A^{*} A \geqq A A^{*}$. Thus $\left(A^{*} A^{2} x, x\right)=0$, which implies that

$$
\left(A^{*} A x, A^{*} A x\right)=\left\|A^{*} A x\right\|^{2}=0 .
$$

Hence $A^{*} A x=0$. But

$$
0=\left(A^{*} A x, x\right)=(A x, A x)=\|A x\|^{2},
$$

implies that $x \in N(A)$, or $N\left(A^{2}\right) \subseteq N(A)$. Thus the ascent is 0 or 1 .

Corollary 2. If $A$ is hyponormal, then $\omega(A)$ consists of $\sigma(A)$ except for poles of the resolvent of finite rank.

Proof. Since $A$ is hyponormal, $\lambda-A$ is hyponormal for all $\lambda$. Hence, $\alpha(\lambda-A)$ is finite for all $\lambda$. Theorem 1 now gives the desired conclusion.

The connection between Corollary 2 and Coburn's generalization 
of the theorem of Weyl is given in Lemma 2. A point $\lambda \in \sigma(T)$ has finite multiplicity if $n(\lambda-T)<\infty$.

Lemma 2. Let $T$ be a hyponormal operator. An isolated point of $\sigma(T)$ of finite multiplicity is a pole of the resolvent of finite rank.

Proof. In the proof of Theorem 3.1 of Coburn [1], it is shown that if $\lambda$ is an isolated point of finite multiplicity of a hyponormal operator, then $k(\lambda-T)=0$. Now Theorem 9.6 of Taylor [5] implies that $\lambda$ is a pole of the resolvent of finite rank.

Let $\phi$ be a continuous isomorphism of a Banach space $X$ into a Hilbert space $H$ such that $\phi(X)$ is dense in $H$. A bounded linear operator $T$ on $X$ is said to be symmetrizable if the induced operator $\hat{T} \phi(x)=\phi(T x)$ is symmetric with domain $\phi(X)$. The result analogous to Nieto's [2] theorem is immediate after the lemma.

Lemma 3. Let $T$ be a linear operator on $X$. Suppose $T$ is symmetrizable. Then if $\operatorname{Im} \lambda \neq 0$, then $\alpha(\lambda-T)=0$ or 1 .

Proof. Denote the norm in $H$ by $\|\cdot\|_{H}$. For a symmetric operator $S$ on $H$, Kato [2], p. 270, shows that for $u$ in the domain of $S$

$$
\|(\lambda-S) u\|_{H}^{2}=\|(\operatorname{Re} \lambda-S) u\|_{H}^{2}+(\operatorname{Im} \lambda)^{2}\|u\|_{H}^{2} .
$$

Consequently, if $(\lambda-T)^{2} x=0$, let $u=\phi((\lambda-T) x)$ and $S=\hat{T}$.

Then $(\lambda-S) u=\phi\left((\lambda-T)^{2} x\right)=0$. The above equality implies $\operatorname{Im} \lambda \neq 0$, that $\|\phi[(\lambda-T) x]\|_{H}=0$, or $(\lambda-T) x=0$, since $\phi$ is an isomorphism.

COROLlary 3. If $T$ is symmetrizable, then $\omega(T)$ consists of $\sigma(T)$ except for poles of the resolvent of finite rank.

\section{REFERENCES}

1. L. Coburn, Weyl's theorem for non-normal operators, Michigan Math. J. 13 (1967), 285-288.

2. Tosio Kato, Perturbation theory for linear operators, Springer-Verlag, New York, 1966.

3. J. Nieto, On the essential spectrum of multiplication operators, singular integral operators and symmetrizable operators, Notices Amer Math. Soc. 14 (1967), 671.

4. M. Schechter, On the essential spectrum of an arbitrary operator. I, J. Math. Anal. Appl. 13 (1966), 205-215.

5. A. E. Taylor, Theorem on ascent, descent, nullity and defect of linear operators, Math. Ann. 163 (1966), 18-49.

6. - Introduction to functional analysis, Wiley, New York, 1958.

7. H. Weyl, Uber beschränte quadratische Formen, deren Differenz vollstetig ist, Rend. Circ. Mat. Palermo 27 (1909), 373-392.

California State College, long Beach 